\title{
Experimental study of heat transfer and pressure drop in micro-channel based heat sinks with tip clearance
}

\author{
M. Reyes , J.R. Arias , A. Velazquez , J.M. Vega \\ Aerospace Propulsion and Fluid Mechanics Department, School of Aeronautics, Universidad Politecnica de Madrid, Plaza del Cardenal Cisneros 3, 28040 Madrid, Spain \\ Applied Mathematics Department, School of Aeronautics, Universidad Politecnica de Madrid, Plaza del Cardenal Cisneros 3, 28040 Madrid, Spain
}

A R T I C L E I N F O

Keywords:

Micro-heat sink

Tip clearance

Pressure drop

\begin{abstract}
A B S T R A C T
This article presents an experimental study on the optimisation of micro-heat sink configurations when both thermal effects and pressure drop are accounted for. The interest of the latter is that the practical engineering viability of some of these systems also depends on the required pumping power. The working fluid was water and, according to typical power dissipation and system size requirements, the considered fluid regime was either laminar or transitional, and not fully developed from the hydrodynamics point of view. Five configurations were considered: a reference geometry (selected for comparison purposes) made up of square section micro-channels, and four alternative configurations that involved the presence of a variable tip clearance in the design. The performance of the different configurations was compared with regard to both cooling efficiency and pressure drop. Finally, we also provide some practical guidelines for the engineering design of these types of systems.
\end{abstract}

\section{Introduction}

During the past few years, a large research effort has been devoted to the study of micro-heat sinks. The reason is that practical application of these micro devices is expected to have a significant impact in quite a number of industrial sectors; see Yoo [1], Hassan et al. [2], and Obot [3] for comprehensive reviews in this field. Concerning engineering applications, it is to be noted that engineering products are seldom designed having just one objective in mind. Most often, the industrial viability of a given product depends on whether a compromise has been reached between conflicting objectives. For example, a good technical performance does not guarantee market acceptance unless cost is competitive as well. In the field of micro-heat sinks, the main emphasis has been traditionally placed on the thermal performance of the system, although there are other issues that influence viability. One of these is the pressure drop, which affects both the power required by the pump and the weight and size of the device. These are quite relevant in, e.g., the aerospace sector, where micro-heat-sink devices are increasingly used to control temperature in onboard avionics. Decreasing weight has a multiplicative effect on reducing fuel consumption, and the increasing space limitations in both the cockpit and the avionics bay impose strong constrains to the size of the various on board devices. Then, it could be said that thermal efficiency (namely, the total heat that must be evacuated per unit time) is a natural requirement but pressure drop is a strong design constrain to be reckoned with. For example, in modern fighter aircraft designs in which micro-heat sink devices are used to cool electronics systems (like radar) that dissipate a large amount of power, the onboard fluid management system provides a fixed flow rate of cooling fluid with a prescribed pressure drop. Therefore, it is important to minimise the local pressure drop associated to the different micro-cooling devices.

A comprehensive review of the literature dealing with heat sink optimisation with regard to heat transfer and pressure drop appears in the introduction of a recent article published by Khan et al. [4]. In this introduction, the authors stress the importance of accounting with these two effects when practical engineering applications are foresighted. In particular, in the technical chapters, the authors numerically asses combined thermal resistance and pressure drop behaviour when optimising a heat sink accounting for channel aspect ratio, fin spacing ratio, heat sink material, and Knudsen number. Optimisation of micro-channel heat sinks has also been addressed by Kim and Kim [5] using asymptotic solutions for velocity and temperature distributions. The authors focussed on the case of high channel aspect ratio (height/width $>4$ ), high ratio of solid to fluid thermal conductivity $(>20)$, and low Reynolds number ( $<690$ based on the channel hydraulic diameter). In this regime, they provided closed form correlations that relate geometry to heat transfer and pressure drop (pumping power). It was reported that, according to the analysis, optimum thickness of the wall separating channels depends on channel height and solid and 


\begin{tabular}{|ll|}
\hline \multicolumn{2}{|l|}{ Nomenclature } \\
Latin symbols \\
$B$ & channel height \\
$C$ & channel width \\
$G$ & volume flow rate $(1 /$ min) \\
$P_{\mathrm{P}}$ & pumping power $(\mathrm{W})$ \\
$Q$ & heat transfer rate $(\mathrm{W})$ \\
$Q_{S}$ & heat transfer rate per unit platform area $\left(\mathrm{W} / \mathrm{cm}^{2}\right)$ \\
$R e$ & Reynolds number based on hydraulic diameter of \\
& the inlet channel, average inlet velocity, and \\
$t_{C}$ & viscosity measured at the inlet temperature \\
Greek symbols & convective hydrodynamic time \\
$\Delta P$ & pressure drop (Pa) \\
$\mu$ & fluid viscosity \\
\hline
\end{tabular}

fluid thermal conductivities, but not on pumping power, fluid viscosity and micro-channel length. On the contrary, optimum channel width is a function of fluid and solid properties and pumping power. Micro-heat sink optimisation has been also considered by Husain and Kim [6], who used an evolutionary algorithm for optimisation purposes, and defined an objective function depending on both heat transfer and pumping power. In particular, they choose to optimise two design variables: wall thickness and channel width, and found that a clearly defined Pareto front exists. This fact suggests that, in their problem, there is a trade-off between thermal resistance and pumping power on the selected space of design parameters. Foli et al. [7] and Ryu et al. [8] followed a somewhat similar approach but, instead, they used the pumping power as a constrain in the optimisation algorithms. A very detailed experimental study on the pressure drop and heat transfer in a micro-channel has been published by Qu and Mudawar [9], who considered an array of rectangular micro-channels 231 microns wide and 713 microns deep in the Reynolds number span from 139 to 1672 , for two different heat fluxes: 100 and $200 \mathrm{~W} / \mathrm{cm}^{2}$. They provided an interesting set of conclusions. Namely: a) contrary to what other articles have suggested, the conventional Navier-Stokes equations adequately predict fluid flow and heat transfer behaviour inside micro-channel heat sinks; b) early laminar to turbulent transition, also reported in other papers, was not observed in the range up to Reynolds number equal to 1672 ; c) higher Reynolds number are beneficial for the heat transfer standpoint at the expense of a greater pressure drop; and d) the channel top wall, made up of polycarbonate plastic, can be considered as adiabatic for all practical purposes.

The pressure drop in a micro-channel depends on a number of factors. For example, Croce et al. [10] have reported a significant influence of surface roughness on pressured drop and provided correlations among the Nusselt and Reynolds numbers, the friction factor, and various geometry parameters; this study was numerical and surface roughness was modelled as set of 3-D conical shapes distributed over a smooth surface. On the other hand, Pence [11] has reported on the use of fractal-like channel networks to reduce the pumping power. In particular, this author stated that, according to her analytical study, if the remaining parameters such as wall temperature and total length of the micro-channel network are kept constant, the use of fractal-like set-ups yields a pressure drop that is of the order of $60 \%$ of the one obtained when using conventional configurations.
In this context of devising methods to reduce the pressure drop while keeping a reasonable thermal performance, a relatively simple approach consists of using the tip clearance as a control parameter. It is clear that the resulting flow bypass affects the thermal performance of the system but the overall effect (pressure drop plus heat transfer) might be favourable. This physical effect has long been considered and related studies have been published in the specialised literature. Sparrow et al. [12], back in the seventies, presented an analytical study on the laminar heat transfer associated to shrouded thin arrays where they concluded that conventional uniform heat transfer coefficient models are not applicable to this type of configuration. An experimental study including the same type of geometry was published by Sparrow and Kaddle [13]. The authors considered air as the cooling fluid and the flow was turbulent. The reported that for clearances in the range $10-30 \%$ of the fin height, the heat transfer coefficients were $85-64 \%$ of those for the zero clearance case, and that the ratio of the with-clearance to no-clearance heat transfer coefficient was a function of only the clearance-to-fin-height ratio, independent of both the flow rate and fin height. Also, it was mentioned that the presence of clearance slowed the rate of thermal development of the flow. It is to be noted that no information about pressure drop was provided in references $[12,13]$. Similar experimental correlations were provided by Wirth et al. [14] for air cooling devices in the case when flow bypass is present. Development of a new semianalytical model for the accurate prediction of pressure losses in configurations exhibiting bypass has been reported by Coetzer and Visser [15]. A specific study on the effect of tip clearance on the cooling performance of an array of micro-channels for a fixed pumping power bounding condition has been reported by Min et al. [16], who used a numerical model under the assumption of fully developed laminar flow. The conclusion was that for any prescribed pumping power there exists an optimum tip clearance that minimizes thermal resistance. Also, it is noteworthy to mention the work by Dogruoz et al. [17], Jeng [18], Moores et al. [19], and Rozati et el [20], who considered the effect of tip clearance in micro-heat sinks that use pin fin configurations instead of channels.

The objective of the present article is to perform an experimental study on a series of configurations that involve arrays of micro-channels so as to infer information about what is the specific setup that provides an optimum combination of thermal performance and pumping power. With regard to the organisation of the work to be presented hereafter, the following section deals with the statement of the problem. Next, the experimental test bench and results are described. Finally, conclusions and engineering design guidelines are provided.

\section{Description of the problem and experimental setup}

We have studied the behaviour of five different configurations, using water as the cooling fluid. The basic setup is as shown in Fig. 1,

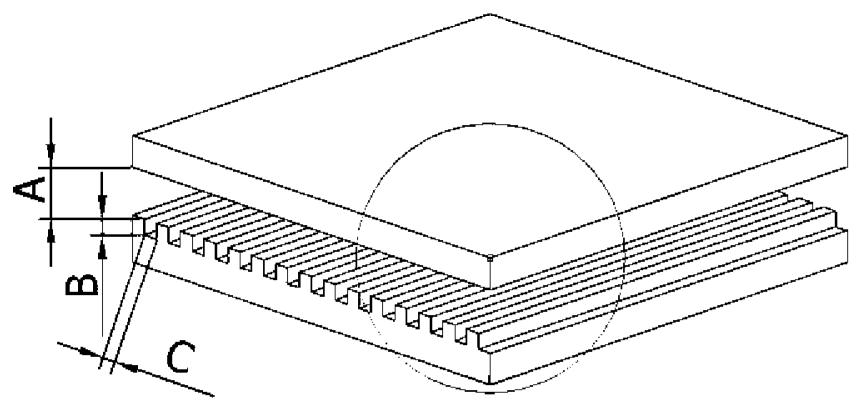

Fig. 1. Generic view of the model setup. 


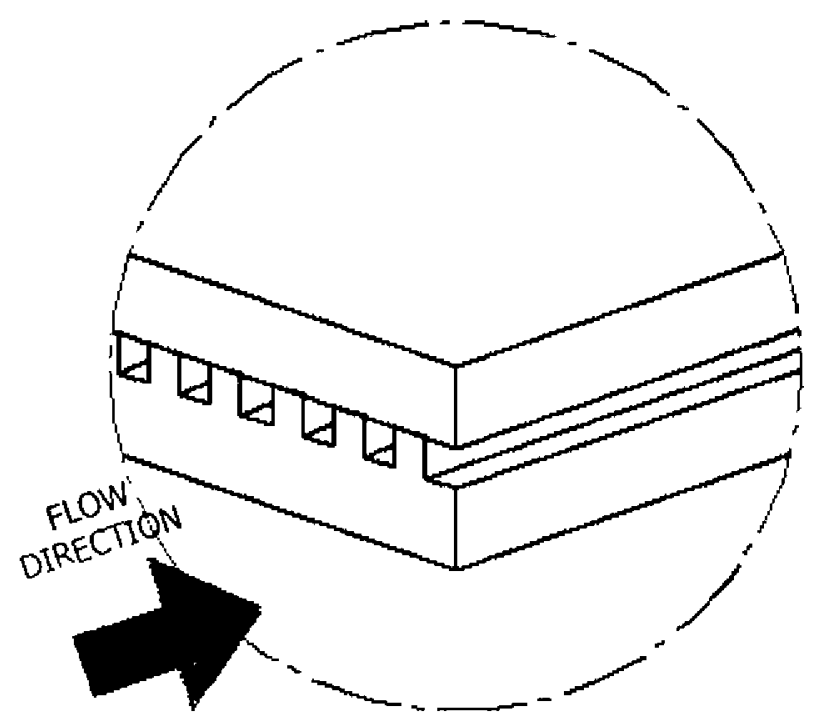

Fig. 2. Close-up view of configuration 1.

with channel height, B, and width, C, equal to $500 \mu \mathrm{m}$ in all cases. The platform on which the micro-channels were manufactured had an area of $15 \mathrm{~mm} \times 15 \mathrm{~mm}$. This means that we had 15 parallel micro-channels whose length was $15 \mathrm{~mm}$ each. The ratio of microchannel length to hydraulic diameter was 30 . This ratio is low if fully developed flow is sought along most of the micro-channel length. For example, if the inlet Reynolds number (based on the channel hydraulic diameter) is of the order of 1000, the entrance length is of the order of $8 \mathrm{~mm}$ (Pelesko and Bernstein [21]), which is half of the micro-channel length, meaning that the flow is not developed in our series of experiments. This is a typical practical situation because industrial applications impose limits on the actual length of micro-channels. For example, when dealing with the cooling of avionics equipments placed inside Arinc-type avionics racks, the maximum allowable dimension of the microcooler is of the order of $10-20 \mathrm{~mm}$. The material of the base where the micro-channels were manufactured was aluminium alloy certified for aeronautics applications while the top plate was manufactured on polycarbonate. The ratio of the thermal conductivity of aluminium alloy to polycarbonate is 850 , meaning that the top plate can be considered as adiabatic.

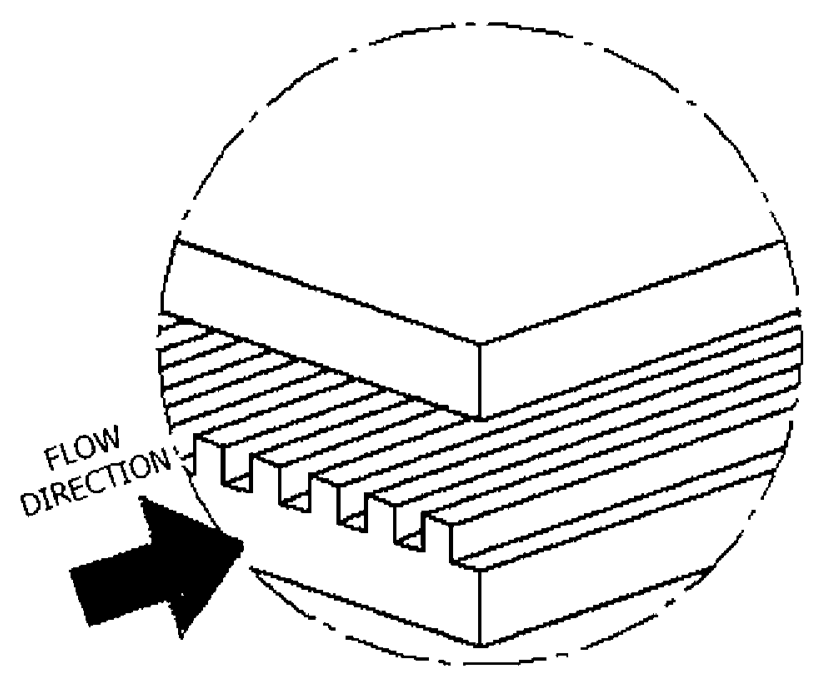

Fig. 3. Close-up view of generic configurations 2,3 , and 4

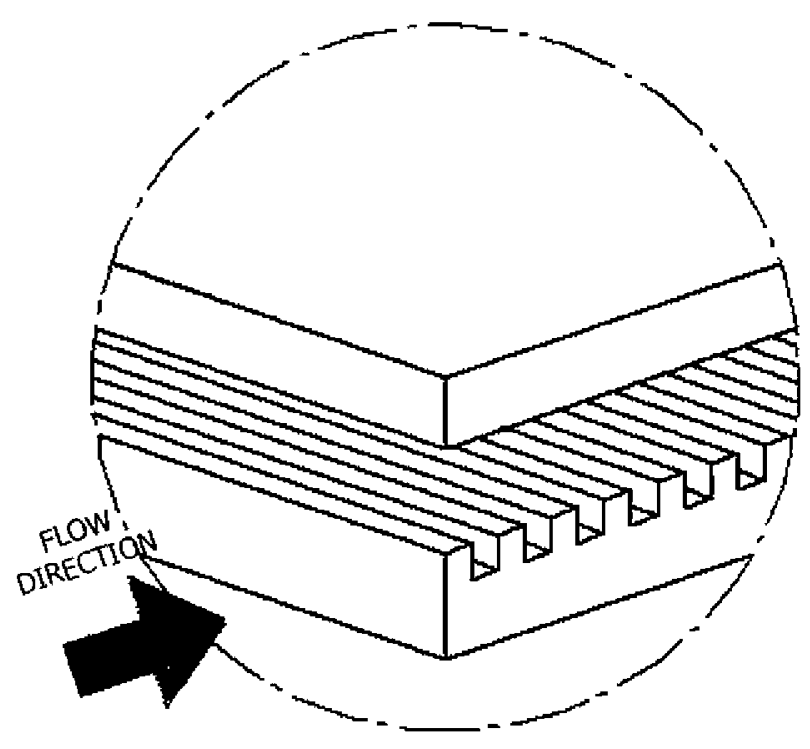

Fig. 4. Close-up view of configuration 5 .

The details of the tested configurations are as follows:

- Configuration 1 (see Fig. 2), which was considered to be the baseline. No tip clearance was allowed and the working fluid flowed parallel along the micro-channels.

- Configurations 2, 3, and 4 (see Fig. 3). The working fluid also moved along the micro-channels but three different tip clearances were allowed: $250 \mu \mathrm{m}, 500 \mu \mathrm{m}$, and $1000 \mu \mathrm{m}$, respectively. These tip clearances represented $50 \%, 100 \%$, and $200 \%$ respectively of the channel height $(500 \mu \mathrm{m})$.

- Configuration 5 (see Fig. 4). The flow motion was perpendicular to the micro-channels and the tip clearance was $500 \mu \mathrm{m}$.

Two stagnation flow chambers (see Fig. 5) were implemented, upstream and downstream of the micro-channels, to distribute the flow. The two chambers were micro-machined on the same base as the micro-channels. The flow of water came in perpendicular to the base. We estimated, using standard correlations [22], that heat transfer associated to these stagnation chambers was of the order of $5 \%$ of the total.

We could have designed thermally insulated stagnation chambers and, also, we could have supplied the cooling flow as being uniform and parallel to the micro-channels. However, we opted for a configuration that is as close as possible to practical industrial applications. What happens is that in these practical applications, because of cost and available space, it is not always possible to use an inlet chamber that fully guaranties flow uniformity. Also, since the flow supply hoses should not be placed close to the base because of the need to pass vibration tests for certification purposes, we preferred to implement a perpendicular supply system (see Fig. 6 below). The inlet and outlet hoses were located at approximately the centre of the stagnation chamber roof and had an internal diameter of $3 \mathrm{~mm}$.

The heating system consisted of a block of aluminium alloy with an insertion of two electrical resistances. In a preliminary set of experiments, the micro-heat sink and the heating system were separate components joined by pressing them together with a constant mechanical force, using thermal grease at the contact surface. However, we encountered difficulties in guaranteeing the repeatability of the experimental series. In particular, the grease film had a tendency to deteriorate over time, probably owning to heat and surface contact pressure. We could have used thermal 

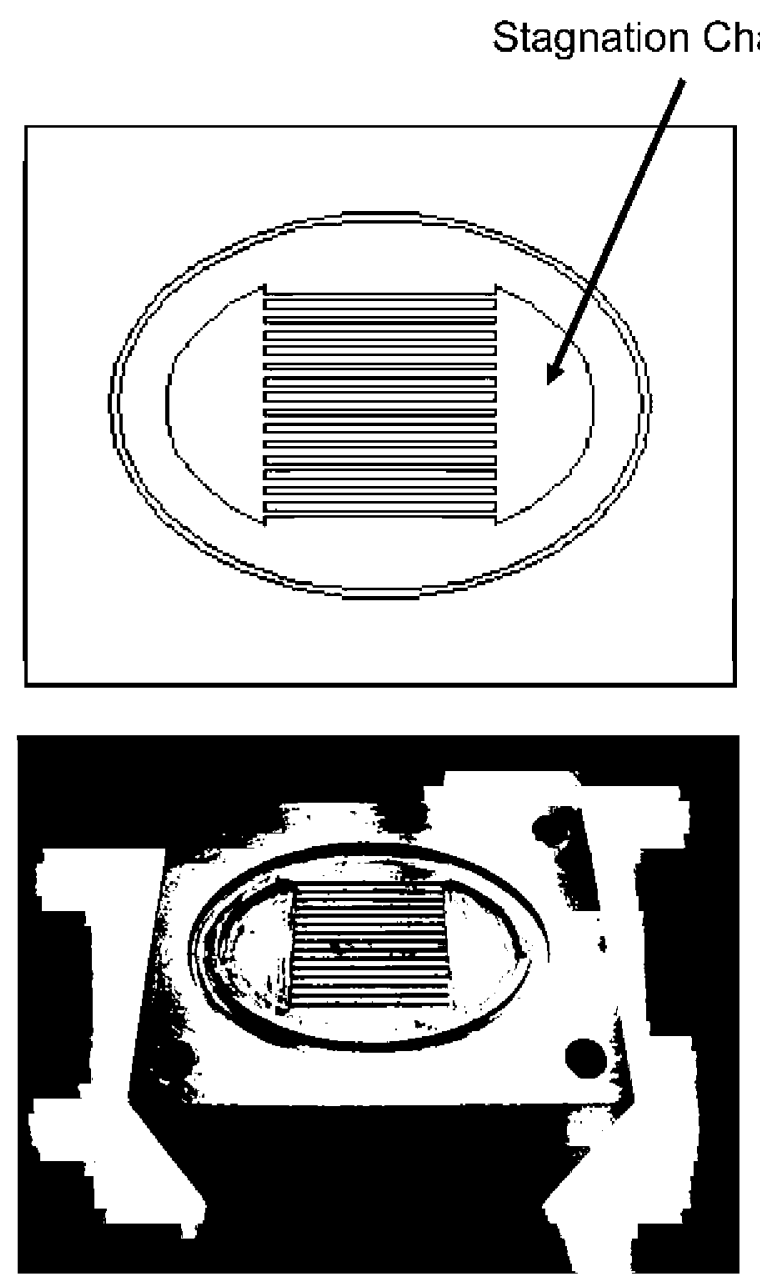

Fig. 5. Schematic (top) and actual (bottom) views of the micro-channels and stagnation chambers in configurations $1-4$.

pads to solve the difficulty, but instead, we decided to manufacture a new set of prototypes on which the heat exchange area was micro-machined directly on top of the heating block, which solved the issue of test's repeatability. Ensuring a good contact between elements is, nevertheless, a critical design aspect that must be dealt

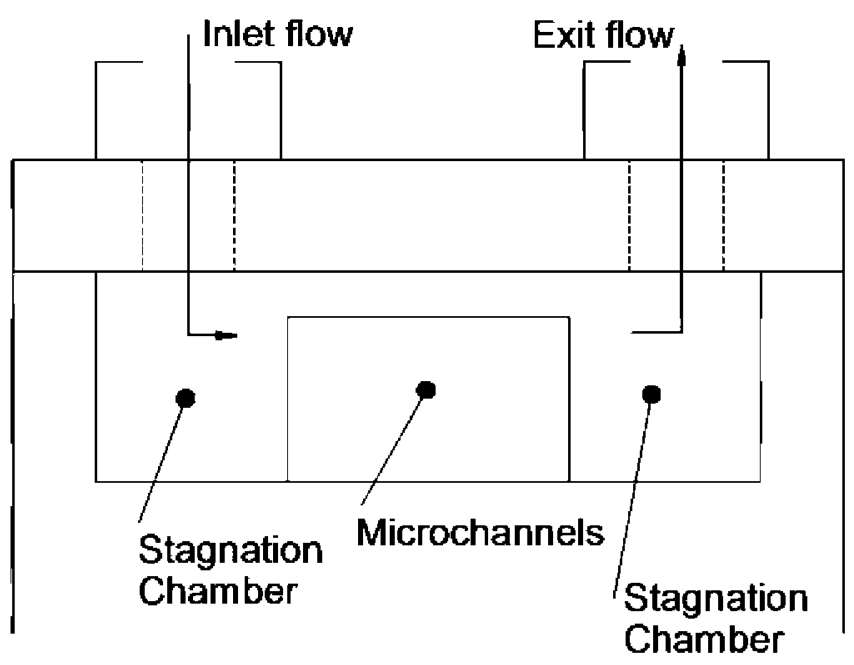

Fig. 6. Lateral view sketch of the experimental setup. with in practical industrial applications. As our experience has shown, integrated systems are superior to those that need additional means to achieve a robust thermal contact. A PID control system was in place to maintain the aluminium wall temperature right below the micro-channels, at $70^{\circ} \mathrm{C}$. Inlet water temperature was regulated to enter the micro-heat sink at $35^{\circ} \mathrm{C}$. An overview of the whole block is presented in Fig. 7.

Two T-type TC-SA thermocouples were inserted right below of the micro-channels at a distance of $1.5 \mathrm{~mm}$ under the surface. Two additional thermocouples were used to measure inlet and outlet flow temperature. We requested to the thermocouples supplier that they all belong to the same manufacturing series so that the measurements errors $\left( \pm 0.5^{\circ}\right)$ were all based in the same direction. Pressure drop was measured by using two pressure sensors (Ellison GS4101) that were located in the inlet and outlet hoses right outside of the stagnation chambers. A flowmeter (GEMS FT110) was placed on the outlet hose. The total mass flux was maintained at a fixed value in each run by the pump itself. The current in the electrical resistance was controlled in such a way that the temperature of the metal base was maintained at the prescribed value. Heat losses from the base were calculated as the heat evacuated by the coolant liquid, which in turn resulted from the total mass flux and the inlet and outlet temperatures, taking into account heat losses in the stagnation chambers (estimated as indicated above). The data acquisition system was a Keithley KUSB 3108. The whole setup was controlled by using PID control system formulated in Labview software. A schematics of the experimental test bench is presented in Fig. 8 .

\section{Results}

The average results for the baseline configuration 1 (no tip clearance) are given in Table 1 . Six different volume flow rates $G$ (litres per minute) were considered in the range from 0.16 to $1.00 \mathrm{l} / \mathrm{min}$. Each of these volume flow rates had an associated

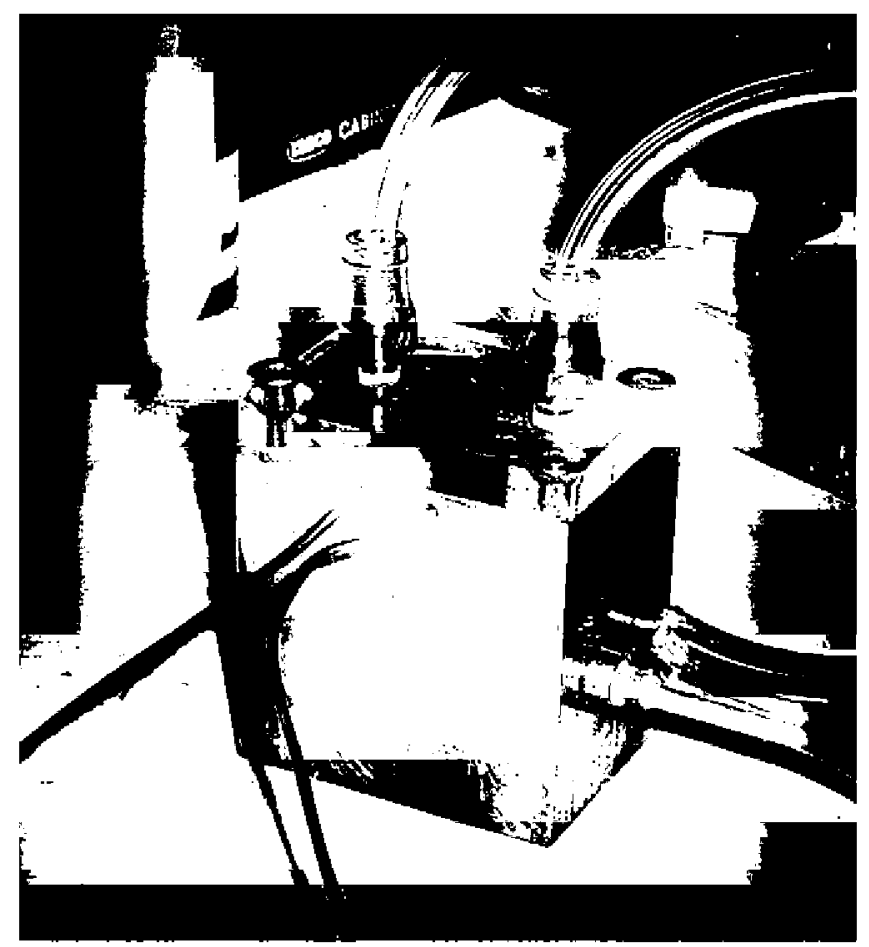

Fig. 7. Overview of the heated block with micro-machined channels, polycarbonate top wall, different insertions and supply and exit hoses. 


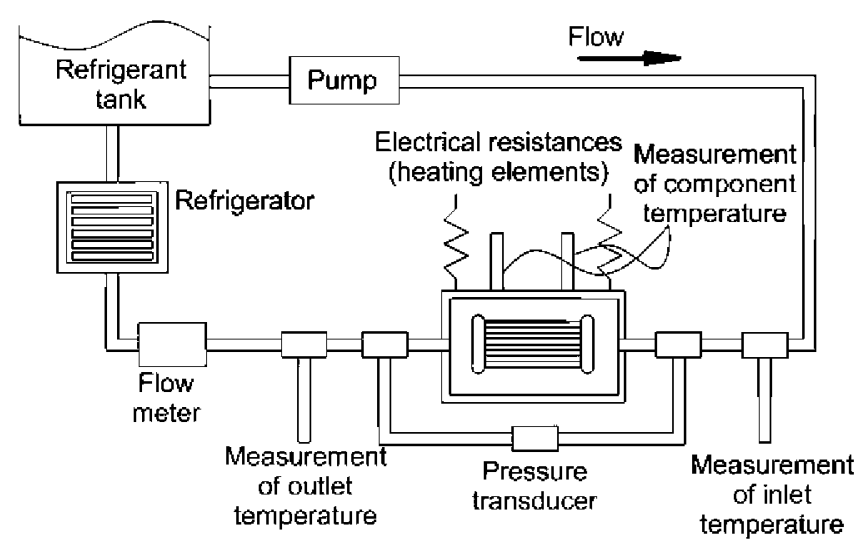

Fig. 8. Schematics of the experimental setup.

Reynolds number, $R e$, based on the average inlet velocity and hydraulic diameter of the micro-channels. $Q$ is the evacuated heat per unit time, $Q_{S}$ is the ratio of $Q$ to the platform area of the microheat sink $\left(15 \times 15 \mathrm{~mm}=225 \mathrm{~mm}^{2}=2.25 \mathrm{~cm}^{2}\right), \Delta P$ is the pressure drop, and $P_{\mathrm{P}}$ is the pumping power (volume flow rate times $\Delta P$ ). Thus, we have basically four laminar (Re 416 to 1300) and two transitional ( $R e 1959$ and 2600) flows. The evacuated heat is in the range from 52 to $121 \mathrm{~W} / \mathrm{cm}^{2}$, which is representative of the foreseen evolution of industrial power electronics dissipation for the next decade or so. The chosen platform area fits the surface of the average heat dissipating electronic component in avionics applications.

Configurations 2-5 are considered in Table 2, where the average results are provided as referred to the results of configuration 1 (the baseline). In other words, $Q^{\prime}, \Delta P^{\prime}$, and $P_{\mathrm{P}}{ }^{\prime}$ are the ratios of evacuated heat, pressure drop, and pumping power in these configurations to their counterparts in the baseline.

The following remarks about the results presented in Table 2 are in order:

- If the volume flow rate is kept constant, implementation of tip clearance always yields less heat transfer than in the baseline configuration, as appreciated in Fig. 9. Depending on the volume flow rate and tip clearance height, the ensuing heat transfer ranges from $65 \%$ to $85 \%$ of the baseline heat transfer.

- For a given volume flow rate, the heat transfer does not behave monotonically as the tip clearance is increased in configurations 2-4. Instead, it starts increasing, reaches a peak at some optimum tip clearance height, and goes down again. We think that this effect is associated to the generation of 3-D structures that enhance convective heat transfer and loom over the top of the channel separation walls while simultaneously developing downstream. If the tip clearance is very small, the growth of these 3-D structures is either inhibited or delayed by the close presence of the top horizontal wall. If the tip clearance is very large, the effect of the top wall is small and the flow attains

Table 1

Results of the baseline configuration 1 with no tip clearance.

\begin{tabular}{llllrl}
\hline \multicolumn{6}{l}{ Configuration 1} \\
\hline$G(1 / \mathrm{min})$ & $\mathrm{Re}$ & $\mathrm{Q}(\mathrm{W})$ & \multicolumn{1}{l}{$\mathrm{QS}_{\mathrm{S}}\left(\mathrm{W} / \mathrm{cm}^{2}\right)$} & $\Delta P(\mathrm{~Pa})$ & $P_{\mathrm{P}}(\mathrm{W})$ \\
\hline 0.16 & 416 & 117 & 52 & 4907 & 0.013 \\
0.25 & 650 & 146 & 65 & 7251 & 0.030 \\
0.33 & 858 & 171 & 76 & 10,456 & 0.058 \\
0.50 & 1300 & 221 & 98 & 16,589 & 0.138 \\
0.75 & 1959 & 259 & 115 & 29,916 & 0.373 \\
1.00 & 2600 & 272 & 121 & 55,296 & 0.921 \\
\hline
\end{tabular}

Table 2

Comparison between the results obtained for configurations 2 to 5 and those of configuration 1.

\begin{tabular}{|c|c|c|c|c|c|c|c|c|}
\hline \multirow[t]{2}{*}{$G(1 / \mathrm{min})$} & \multicolumn{2}{|c|}{ Conf. 2} & \multicolumn{2}{|c|}{ Conf. 3} & \multicolumn{2}{|c|}{ Conf. 4} & \multicolumn{2}{|c|}{ Conf. 5} \\
\hline & $Q^{\prime}$ & $\Delta P^{\prime}=P_{\mathrm{P}^{\prime}}^{\prime}$ & $Q^{\prime}$ & $\Delta P^{\prime}=P_{\mathrm{P}}^{\prime}$ & $Q^{\prime}$ & $\Delta P^{\prime}=P_{\mathrm{P}}^{\prime}$ & $Q^{\prime}$ & $\Delta P^{\prime}=P_{\mathrm{P}}^{\prime}$ \\
\hline 0.16 & 0.76 & 0.29 & 0.83 & 0.18 & 0.64 & 0.10 & 0.74 & 0.17 \\
\hline 0.25 & 0.77 & 0.32 & 0.81 & 0.24 & 0.65 & 0.15 & 0.71 & 0.25 \\
\hline 0.33 & 0.81 & 0.31 & 0.83 & 0.30 & 0.69 & 0.23 & 0.70 & 0.33 \\
\hline 0.50 & 0.80 & 0.39 & 0.82 & 0.36 & 0.65 & 0.31 & 0.71 & 0.40 \\
\hline 0.75 & 0.81 & 0.53 & 0.84 & 0.42 & 0.71 & 0.40 & 0.76 & 0.45 \\
\hline 1.00 & 0.85 & 0.50 & 0.88 & 0.40 & 0.75 & 0.40 & 0.78 & 0.45 \\
\hline
\end{tabular}

a quasi 2-D character again with small 3-D effects over the walls that separate the channels. Then, it appears that there should be some optimum tip clearance height that maximises the 3-D effects responsible for promoting heat transfer. This subject, which is still open, is the objective of some of our current research activity.

- If the volume flow rate is kept constant, the tip clearance always leads to a smaller pressure drop and, therefore, to a smaller pumping power. This is appreciated in Fig. 10. Depending on the volume flow rate and tip clearance, the ensuing pumping power is in the range of $10-53 \%$ of the baseline pumping power.

- For a given volume flow rate, the pumping power always decreases monotonically as a function of the tip clearance.

- The use of micro-channels perpendicular to the flow direction (configuration 5) could also be an acceptable compromise between heat transfer and pressure drop. However, for the same tip clearance height, the results are consistently better when the micro-channels are aligned to the flow (configuration 3 ).

- When looking for a reasonable design point for an actual thermal control system, it is important to account for the fact that neither the heat transfer nor the pressure drop behave linearly as a function of the volume flow rate. Even in the case of the baseline configuration, the fact that a significant portion of the flow is non-developed (owing to the short channel length) means that the laminar Nusselt number is not constant, which is appreciated in Figs. 9 and 10.

- When considering the combined behaviour of heat transfer and pressure drop, the most favourable results are obtained with the lower volume flow rates. For example, configuration 3 (tip clearance height equal to $500 \mu \mathrm{m}$ for a channel height of $500 \mu \mathrm{m}$ ) with the lowest volume flow rate of $0.16 \mathrm{l} / \mathrm{min}$ (equivalent to $R e=416$ in the baseline configuration) yields a heat transfer that is $83 \%$ of the baseline configuration while the pumping power has been reduced to only $18 \%$ of the baseline (it has been divided by a factor of 5.5). In this situation, the heat transfer is $43 \mathrm{~W} / \mathrm{cm}^{2}$, which still represents a large improvement over conventional plates of fins used for thermal

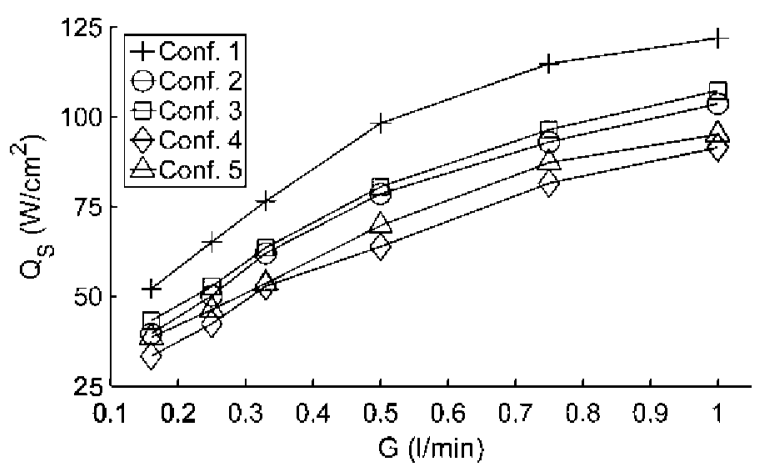

Fig. 9. Heat transfer in configurations $1-5$ vs. the volume flow rate. 


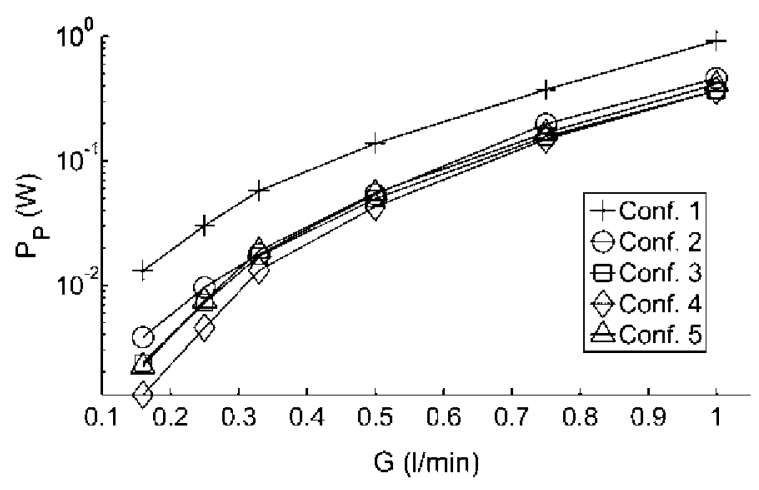

Fig. 10. Pumping power in configurations $1-5$ vs. the volume flow rate.

control purposes of avionics equipment. In these conventional systems, the typical heat transfer rate at the component level is in the range from 2 to $5 \mathrm{~W} / \mathrm{cm}^{2}$. When considering the same configuration at a higher volume flow rate $(0.50 \mathrm{l} / \mathrm{min}$, equivalent to $R e=1300$ in the baseline configuration), the heat transfer is $83 \%$ of the baseline configuration while the pumping power has been divided by a factor of 3.3. In this case, the actual heat transfer is $81 \mathrm{~W} / \mathrm{cm}^{2}$. The benefit of the tip clearance is not so clear at the largest flow rate, as appreciated in Fig. 11, where the heat transfer rate is plotted vs. the required pumping power. This is due to the flow topology, which is quasi-two dimensional at the upper part of the channel. A better performance of configurations with a tip clearance at large flow rates would require enhancing three-dimensional convection at the upper region of the channel, which is the object of current research.

- In any event, configuration \#3 outperforms the baseline configuration for thermal efficiencies smaller than $90 \mathrm{~W} / \mathrm{cm}^{2}$ Fig. 11 illustrates well the main advantage of the tip clearance, and the fact that configuration \#3 seems to be the best choice. For instance, for a thermal efficiency of $65 \mathrm{~W} / \mathrm{cm}^{2}$, the pumping powers associated with configurations \#1 and \#3 are 3.1 and $2.0 \mathrm{~W}$, respectively, which means that configuration \#3 requires about $33 \%$ less pumping power than configurations \#1 for the same heat evacuation target.

Regarding the repeatability of the results, we performed three experimental campaigns for each configuration and each flow rate. In each campaign we acquired thirty measurements. The dispersion of experimental heat transfer and pressure drop results for configurations 1 and 3 are presented in Figs. 12 and 13 below, where it is seen that the dispersion in the measured volume flow rate was of the order of $\pm 5 \%$. The actual dispersion of heat transfer

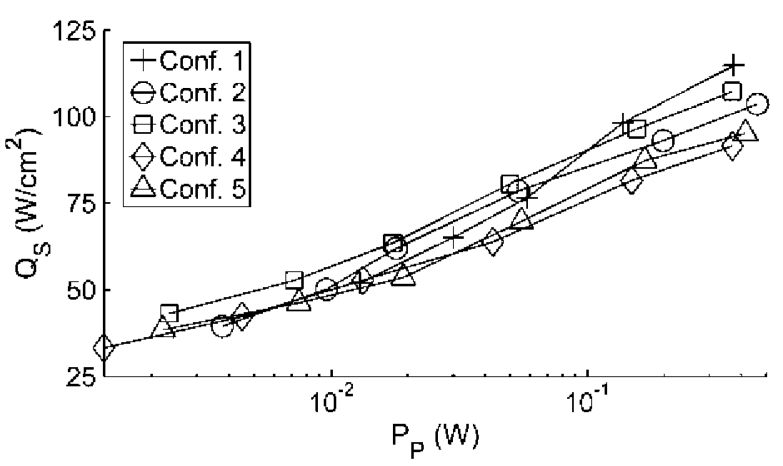

Fig. 11. Heat transfer in configurations $1-5$ vs. the pumping power.

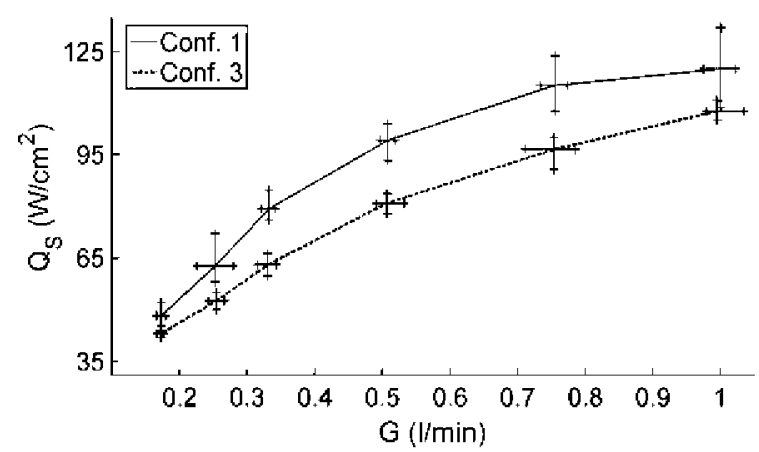

Fig. 12. Dispersion of heat transfer experimental points for configurations 1 and 3 as a function of the volume flow rate.

results around configuration 1 was of the order of $\pm 6 \%$, while it was significantly smaller $( \pm 2.6 \%)$ for configuration 3 and the other configurations, not represented in Fig. 12. A closer look at the time series shows that the larger dispersion in the baseline configuration is associated with unsteady effects, which are enhanced to a larger level in configuration 1 because the effective Reynolds number is larger. The Fourier transform of all time series peaks at a frequency that is slightly larger than that of the data acquisition system, which is $2.5 \mathrm{~Hz}$. This is much smaller than the frequencies associated with relevant unsteady effects, namely the frequency of the volumetric pump, which ranges between 60 and $70 \mathrm{~Hz}$, depending on the volume flow rate, and the inverse of the convective hydrodynamic time, $t_{c}^{-1}=\operatorname{Re} \mu / \mathrm{C}^{2}$ which ranges from 16 to $100 \mathrm{~Hz}$ depending on the Reynolds number. The observed dispersion is seemingly due to hydrodynamic instabilities. This generates local eddies that increase viscous dissipation (and thus, also increase pressure drop) but mainly promote local mixing, instead of the overall transversal convection that would be necessary to increase heat transfer from the hot walls to the bulk.

At this stage, it is worth mentioning the conclusions drawn by Min et al. [16] in their numerical study of a somewhat similar problem. In particular, they found out that for a fixed pumping power, thermal resistance is minimised (the optimum design point) when the ratio of tip clearance to channel width is 0.6 approximately. Our problem was different in the sense that we did not fixed pumping power but measured it (it was not a constrain in our setup) and, also, because the experimental nature of our work did not allowed for a nearly continuous variation of the tip clearance, as it can be done in a numerical study. Nevertheless, we found that our optimum tip clearance (see Table 2) would be somewhere inside the span of $0.5-1.0$ of the ratio of tip clearance to channel width, thereby coinciding qualitatively with the results of Min et al. [16]. The case of pin tip clearance seems to be different for a number of

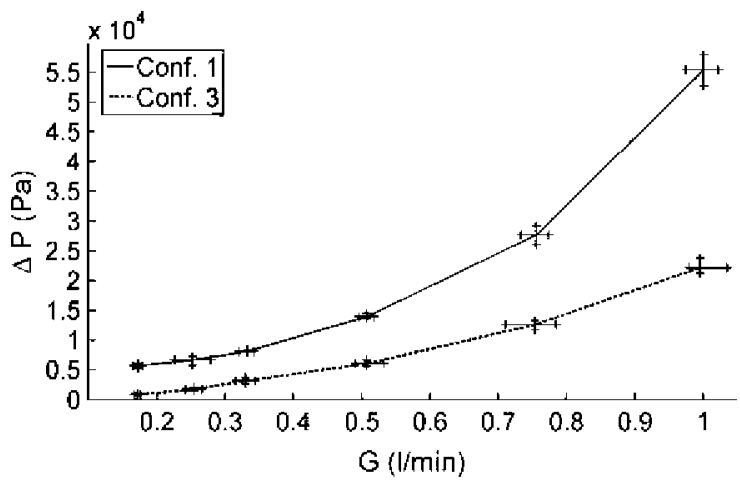

Fig. 13. As in Fig. 12 but regarding the pressure drop. 
reasons that have to do, mostly, with geometry considerations. In this case, Rozati et al. [20] have reported that the optimum tip clearance in the low Reynolds number regime would 0.3 times the pin diameter (somewhat similar to the fin width).

\section{Conclusions}

We have studied the effect of tip clearance on micro-channel flow based thermal control systems when, owing to engineering design restrictions, the flow itself cannot be considered as fully developed. The study has accounted for two parameters of practical interest, namely the heat transfer and the pressure drop (which is related to the pumping power). Four configurations involving a tip clearance have been analysed and compared to a baseline configuration of micro-channel flow without tip clearance. The height of the square section micro-channels was $500 \mu \mathrm{m}$. Tip clearances of $250 \mu \mathrm{m}, 500 \mu \mathrm{m}$, and $1000 \mu \mathrm{m}$ were considered. One additional configuration with the channels perpendicular to the main flow and a tip clearance of $500 \mu \mathrm{m}$ was studied. For each configuration, six different volume flow rates were considered. These flow rates, in the case of the baseline configuration, led to Reynolds numbers in the range from 416 to 2600 .

The main conclusion of the work been presented is that implementation of tip clearance in active micro-channel based thermal control systems is an attractive option form the practical industrial application standpoint owning to two arguments:

- The added manufacturing cost is negligible since most of the manufacturing complexity is associated the micro-machining of the micro-channels, while the top wall can be easily set at a lower or higher height.

- The deterioration in heat transfer caused by the tip clearance is small while the savings in pumping power are large. In our study, for the optimum tip clearance height, the heat transfer (at the lowest volume flow rate, $R e=416$ ) was $83 \%$ of the baseline configuration. However, the required pumping power was only $18 \%$ of the baseline case. The advantage of introducing a tip clearance can also be illustrated noting that the required pumping power can almost be halved maintaining the thermal efficiency. At a larger volume flow rate $(R e=1300)$, the heat transfer behaved similarly while the pumping power was $36 \%$ of the baseline configuration.

Regarding future work, there are three related issues to be analysed: a) the existence of an optimum tip clearance height that, seemingly, has to do with stability issues within the fluid; b) the feasibility of enhancing three-dimensional convection in the tip clearance flow region, which could be done manufacturing some obstacles in the top wall aiming to generate 3-D flow disturbances that promote heat transfer with a limited pressure drop; c) to further understand the unsteady nature of the dispersion of results, which is higher in the baseline configuration than in those with tip clearance.

\section{Acknowledgements}

M. Reyes and A. Velazquez are funded by the Spanish Ministry of Education and Science (Ministerio de Educacion y Ciencia) under research contract DPI2009-07591. J.R. Arias and J.M. Vega are funded by the Subdireccion General de Investigacion of Comunidad de Madrid under research contract S2009/DPI-1572. The authors gratefully acknowledge the support. We are also indebted to an anonymous referee for various useful suggestions on a former version of the paper.

\section{References}

[1] J.Y. Yoo, Recent studies on fluid flow and heat transfer in thermal microdevices, Nanoscale and Microscale Thermophysical Engineering 10 (2006) $67-81$.

[2] I. Hassan, P. Phutthaveng, M. Abdelgamad, Microchannel heat sinks: an overview of the State of the Art, Microscale Thermophysical Engineering 8 (2004) 183-205

[3] N.T. Obot, Towards a better understanding of friction and heat/mass transfer in microchannels - a literature review, Microscale Thermophysical Engineering 6 (2002) 155-173.

[4] W.A. Khan, J.R. Culham, M.M. Yovanivich, Optimization of microchannel heat sinks using entropy generation minimization method, IEEE Transactions on Components and Packaging Technologies 32 (2009) 243-251.

[5] D.K. Kim, S.J. Kim, Closed-form correlations for thermal optimisation of microchannels, International Journal of Heat and Mass Transfer 50 (2007) 5318-5322.

[6] A. Husain, K.Y. Kim, Optimization of microchannel heat sink with temperature dependent fluid properties, Applied Thermal Engineering 28 (2008) 1101-1107.

[7] K. Foli, T. Okate, M. Olhafer, Y. Jin, B. Senhott, Optimisation of a micro heat exchanger: CFD, analytical approach and multi objective evolutionary algorithms, International Journal of Heat and Mass Transfer 49 (2006) 1090-1099.

[8] J.H. Ryu, D.H. Chai, S.J. Kim, Numerical optimisation of the thermal performance of a microchannel heat sink, International Journal of Heat and Mass Transfer 45 (2002) 2823-2827.

[9] W. Qu, I. Mudawar, Experimental and numerical study of pressure drop and heat transfer in a single-phase micro-channel heat sink, International Journal of Heat and Mass Transfer 45 (2002) 2549-2565.

[10] G. Croce, P. D'agara, C. Nonino, Three-dimensional roughness effect on microchannel heat transfer and pressure drop, International Journal of Heat and Mass Transfer 50 (2007) 5249-5259.

[11] D.V. Pence, Reduced pumping power and wall temperature in microchannel heat sinks with fractal-like branching channel networks, Microscale Thermophysical Engineering 6 (2002) 319-330.

[12] F.M. Sparrow, B.R. Baliga, S.V. Patankav, Forced convection heat transfer from a shrouded fin array with and without tip clearance, ASME Journal of Heat Transfer 100 (1978) 572-579.

[13] F.M. Sparrow, D.S. Kaddle, Effect of tip to shroud clearance on turbulent heat transfer from a shrouded, longitudinal fin array, ASME Journal of Heat Transfer 108 (1986) 519-524.

[14] R.A. Wirtz, W. Chen, R. Zhou, Effect of flow bypass on the performance of longitudinal fin heat sinks, Transaction of the ASME Journal of Electronic Packaging 116 (1994) 206-211.

[15] C.B. Cretzer, J.A. Visser, Compact modelling of forced flow in longitudinal fin heat sinks with tip bypass, Journal of Electronic Packaging 125 (2003) 319-324.

[16] J.Y. Min, S.P. Jang, S.J. Kim, Effect of tip clearance on the cooling performance of a micro-channel heat sink, International Journal of Heat and Mass Transfer 47 (2004) 1099-1103.

[17] M.B. Dogruoz, M. Urdaneta, A. Ortega, Experiments and modeling of the hydraulic resistance and heat transfer of in-line square pin heat sinks with top by-pass flow, International Journal of Heat and Mass Transfer 48 (2005) 5058-5071.

[18] T.-M. Jeng, A porous model for the square pin-fin heat sink situated in a rectangular channel with laminar side-bypass flow, International Journal of Heat and Mass Transfer 51 (2008) 2214-2226.

[19] K.A. Moores, J. Kim, Y.K. Joshi, Heat transfer and fluid flow in shrouded pin fin arrays with and without tip clearance, International Journal of Heat and Mass Transfer 52 (2009) 5978-5989.

[20] A. Rozati, D.K. Tafti, N.E. Blackwell, Effect of pin tip clearance on flow and heat transfer at low Reynolds numbers, Journal of Heat Transfer 130 (2008) 071704-1-071704-10.

[21] J.A. Pelesko, D.H. Bernstein, Modelling MEMS and NEMS. Chapman \& Hall/CRC New York, 2003.

[22] F.P. Incropera, P. DeWitt, Introduction to Heat Transfer. John Wiley \& Sons, New York, 1996 